\title{
Does patient selection account for the perceived cost savings in outpatient spine surgery? A meta-analysis of current evidence and analysis from an administrative database
}

Presented at the 2018 AANS/CNS Joint Section on Disorders of the Spine and Peripheral Nerves

\author{
Benjamin F. Mundell, PhD, ${ }^{1,2}$ Marcus J. Gates, MD,, ${ }^{2,3}$ Panagiotis Kerezoudis, MD, MS, ${ }^{2,3}$ \\ Mohammed Ali Alvi, MD, ${ }^{2,3}$ Brett A. Freedman, MD, ${ }^{4}$ Ahmad Nassr, MD, ${ }^{4}$ \\ Samuel F. Hohmann, PhD, ${ }^{5,6}$ and Mohamad Bydon, MD2,3
}

\begin{abstract}
${ }^{1}$ Mayo Clinic School of Medicine, ${ }^{2}$ Mayo Clinic Neuro-Informatics Laboratory, and Departments of ${ }^{3}$ Neurosurgery and ${ }^{4}$ Orthopedic Surgery, Mayo Clinic, Rochester, Minnesota; ${ }^{5}$ Center for Advanced Analytics, Vizient Inc., Irving, Texas; and ${ }^{6}$ Department of Health Systems Management, Rush University, Chicago, Illinois
\end{abstract}

\begin{abstract}
OBJECTIVE From 1994 to 2006 outpatient spinal surgery increased 5 -fold. The perceived cost savings with outcomes comparable to or better than those achieved with inpatient admission for the same procedures are desirable in an era where health expenditures are scrutinized. The increase in outpatient spine surgery is also driven by the proliferation of ambulatory surgery centers. In this study, the authors hypothesized that the total savings in outpatient spine surgery is largely driven by patient selection and biases toward healthier patients.

METHODS A meta-analysis assessed patient selection factors and outcomes associated with outpatient spine procedures. Pooled odds ratios and mean differences were calculated using a Bayesian random-effects model. The authors extended this analysis in a novel way by using the results of the meta-analysis to examine cost data from an administrative database of academically affiliated hospitals. A Bayesian approach with priors informed by the meta-analysis was used to compare costs for inpatient and outpatient performance of anterior cervical discectomy and fusion (ACDF) and lumbar laminectomy.
\end{abstract}

RESULTS Sixteen studies with a total of 370,195 patients met the inclusion criteria. Outpatient procedures were associated with younger patient age (mean difference [MD] $-2.34,95 \%$ credible interval [Crl] -4.39 to -0.34 ) and no diabetes diagnosis (odds ratio [OR] 0.78, 95\% Crl 0.54-0.97). Outpatient procedures were associated with a lower likelihood of reoperation (OR 0.42, 95\% Crl 0.16-0.80), 30-day readmission (OR 0.39, 95\% Crl 0.16-0.74), and complications (OR $0.29,95 \% \mathrm{Crl} 0.15-0.50)$ and with lower overall costs (MD $-\$ 121,392.72,95 \% \mathrm{Crl}-\$ 216,824.81$ to $-\$ 23,632.92)$. Additional analysis of the national administrative data revealed more modest cost savings than those found in the meta-analysis for outpatient spine surgeries relative to inpatient spine surgeries. Estimated cost savings for both younger patients ( $\$ 555$ for those age 30-35 years [95\% Crl $-\$ 733$ to $-\$ 374]$ ) and older patients ( $\$ 7290$ for those age $65-70$ years [95\% $\mathrm{Crl}-\$ 7380$ to $-\$ 7190]$ ) were less than the overall cost savings found in the meta-analysis.

CONCLUSIONS Compared to inpatient spine surgery, outpatient spine surgery was associated with better short-term outcomes and an initial reduction in direct costs. A selection bias for outpatient procedures toward younger, healthier patients may confound these results. The additional analysis of the national database suggests that cost savings in the outpatient setting may be less than previously reported and a result of outpatient procedures being offered more frequently to younger and healthier individuals.

https://thejns.org/doi/abs/10.3171/2018.4.SPINE1864

KEYWORDS spine surgery; inpatient; outpatient; ambulatory surgery; cost analysis; patient selection

ABBREVIATIONS ACDF = anterior cervical discectomy and fusion; $\mathrm{BMI}=$ body mass index; $\mathrm{CCl}=\mathrm{Charlson}$ Comorbidity Index; $\mathrm{CDB} / \mathrm{RM}=($ Vizient $)$ Clinical Data Base/ Resource Manager; $\mathrm{Crl}=$ credible interval; $\mathrm{DM}=$ diabetes mellitus; $\mathrm{LOS}=$ length of stay; $\mathrm{MD}=$ mean difference; OR = odds ratio.

SUBMITTED January 15, 2018. ACCEPTED April 18, 2018.

INCLUDE WHEN CITING Published online September 14, 2018; DOI: 10.3171/2018.4.SPINE1864. 
$\mathrm{O}$ VER the past 2 decades, spine surgical procedures have been increasingly performed in the outpatient setting. From 1994 to 2006 the utilization of outpatient fusions, laminectomies, and discectomies increased over 5-fold. ${ }^{7}$ This is partly due to the perception that outpatient spine surgeries are less costly than similar inpatient procedures., ${ }^{2,7}$ With the increasing scrutiny of health expenditures, the perceived cost savings and comparable outcomes to inpatient procedures have made outpatient spine procedures desirable for payers and surgeons. ${ }^{3}$

Several studies have looked at patient selection and selected outcomes for specific spine surgical procedures, yet no review exists that examines the common patient selection factors and outcomes across the portfolio of outpatient spinal procedures. . $^{3,13,16,22}$ All of these studies report improved outcomes for outpatient surgeries and reduced costs. In this paper the authors hypothesize that patient selection-outpatient spine surgery candidates tend to be younger and healthier-is responsible for the claimed cost savings from outpatient spine surgery. As the demands to conserve healthcare resources increase, it is important for clinicians to understand the array of spine surgical procedures that can be efficiently performed in the outpatient setting. Using available observational studies reporting both inpatient and outpatient outcomes for spine surgery, we performed a meta-analysis to characterize the patient selection factors and outcomes associated with the portfolio of outpatient spine surgery. We extended this analysis in a novel way by using the results of the meta-analysis to examine cost data from a national database of academically affiliated hospitals using a Bayesian modeling approach.

\section{Methods}

\section{Search Strategy}

To qualify the patient selection factors and outcomes for all types of outpatient spinal surgery, a systematic review of the literature available through July 17, 2016, was performed. "Outpatient surgery" was defined as surgery with discharge from the hospital or surgical care setting on the same day of surgery, while "inpatient" surgery was used to describe all surgeries for which the postoperative admission resulted in a dismissal no earlier than the next calendar day. Searches were performed in PubMed, MEDLINE, and Embase for studies comparing selection factors and outcomes for outpatient and inpatient spine surgeries. We used the following Boolean search string (shown in MEDLINE/Embase format): [(outpatient or ambulatory) and (spine or spinal or lumbar or cervical or thoracic or discectomy or laminectomy) and (surgery or operation or procedure or fusion)].ti,ab. The initial search yielded 5817 unique studies. Two independent reviewers (B.F.M. and P.K.) examined the results. Studies not reporting selection factors and outcomes consistently across both cohorts were excluded.

\section{Selection Criteria}

Only studies, including conference presentations and abstracts, reporting both outpatient and inpatient spine surgery patient criteria and outcomes were included in the meta-analysis. Any study reporting at least 1 patient selection factor (e.g., age or sex) and 1 outcome (e.g., cost or 30-day readmission rate) was included. Measures reported for 1 group of patients needed to be reported for the corresponding comparison group to be included in our analysis. Studies reporting outcomes for only inpatient or outpatient spine surgery procedures were excluded, encompassing case series and case reports. Due to the unique nature of the US healthcare system when it comes to the cost of care, only studies looking at surgeries performed in the US were included.

Two reviewers (B.F.M. and P.K.) independently reviewed the search results to determine study inclusion and subsequently abstracted the data. Any disagreements were resolved via consensus between the 2 reviewers. All authors agreed on the final set of studies included and subsequent analysis and conclusions.

\section{Data Extraction}

The data extracted by the 2 aforementioned reviewers included study design, surgery type, number of inpatient and outpatient procedures covered, demographics (to include patient age and sex), measures of comorbidities (both individual and indices-i.e., Charlson Comorbidity Index $[\mathrm{CCI}])$, functional outcomes, readmission rates, reoperation rates, complication rates (including mortality), and reported costs. When mean values and standard deviations were not reported, estimations were made if possible based on available graphs and published methodologies. ${ }^{11}$ Primary data extraction was completed by one reviewer with accuracy of data entry confirmed by the second reviewer. Publication bias was assessed using funnel plots. We observed a variable amount of evidence for publication balance in studies reporting cost differences. For example, cost data appeared to be subject to publication bias, but the number of studies was also small, making this bias difficult to assess using funnel plots.

\section{Cost Data}

Direct cost data on anterior cervical discectomy and fusion (ACDF; ICD-9 code 81.02 and CPT code 22551) and lumbar laminectomy (ICD-9 code 03.09 and CPT codes 63005 and 63047) for patients age 30-35 years and 65-70 years were extracted from a national database of academically affiliated hospitals, the Vizient Clinical Data Base/ Resource Manager (CDB/RM). The Vizient cost data are standardized cost data created using each facility's Medicare cost report cost-to-charge ratio that allows for comparing costs across facilities. All inpatient and outpatient ACDF procedures and lumbar laminectomies performed between October 2012 and September 2016 were included. The data were stratified by age in order to explore the differences in costs for younger and older patients, as younger patients, on average, tend to have fewer comorbidities and are perceived to be healthier. Therefore, younger patients should be less susceptible to patient selection criteria when determining surgical setting, leading to little difference in cost between the inpatient and outpatient settings for this group. 


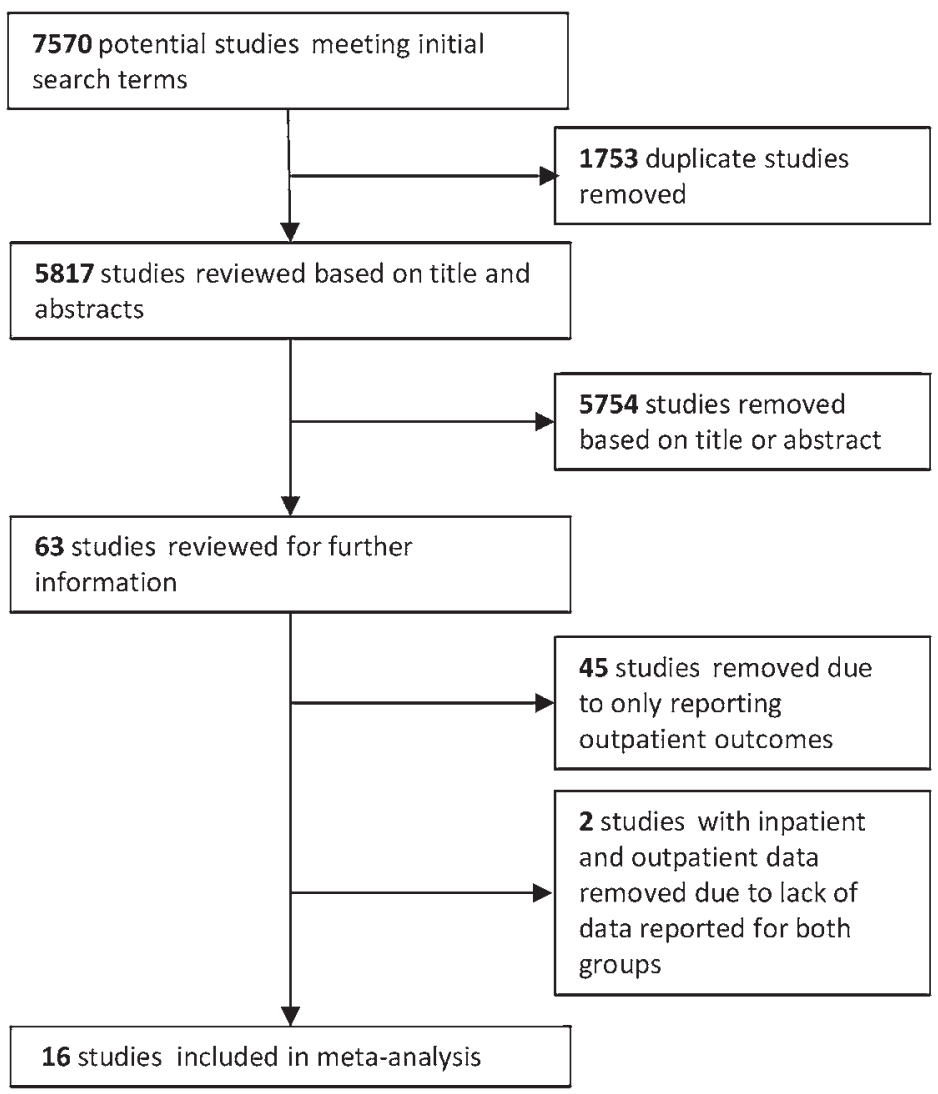

FIG. 1. Study selection flowchart.

\section{Statistical Analysis}

Following data abstraction from the selected articles, a meta-analysis of selection factors and outcomes associated with outpatient procedures was performed. Odds ratios or mean differences were reported for parameters of interest where appropriate.

Due to the potential heterogeneity of the data based on varying study designs and populations, the meta-analysis utilized a Bayesian hierarchical framework to generate a random-effects model for the total pooled effect of each measure of interest. Bayesian analytical approaches have been increasingly used in medical research as they allow researchers to easily incorporate prior knowledge into the modeling approach; past experiences can be easily incorporated in the parameter estimates. The Bayesian approach to meta-analysis allowed the uncertainty associated with each parameter of interest, in this case the patient selection factor and outcome differences, to be explicitly modeled through assumptions about the statistical distribution of these parameters. ${ }^{26}$ Using this modeling approach acknowledges the uncertainty inherent in estimating population parameters from a subset of data but offers the analysts the ability to incorporate some prior knowledge of how the parameters may be statistically distributed.

The CDB/RM cost data were analyzed using a Bayesian estimation for mean differences. This methodology allowed us to use the information gained in the metaanalysis, specifically the distribution of cost differences as determined by the meta-analysis, to inform the estimate of the distribution of cost differences seen in the CDB/ $\mathrm{RM}$ data. An additional advantage over the classic t-test is the ability to accept a null value, in this case that the cost difference found in the meta-analysis persists in the CDB/ RM data. ${ }^{12}$ The Bayesian methodology relies on multiple simulations of the distribution, in this case 100,000 simulations, leading to a robust estimate of the distribution of the mean difference in the $\mathrm{CDB} / \mathrm{RM}$ data. We also ran the model with a naïve prior distribution, a normal distribution with a variance that is 1000 times the standard deviation of the population data, to test the sensitivity of the model to the informed prior distribution. To the best of our knowledge, this is a novel approach for validating reported differences in outcomes from a meta-analysis comparing options for surgical management of a disease.

Data analysis was performed using $\mathrm{R}$ (R Foundation for Statistical Computing, https://cran.r-project.org/ doc/manuals/fullrefman.pdf), the bmeta package (http:// statistica.it/gianluca/bmeta/bmeta_manual.pdf), and the BEST package (https://cran.r-project.org/web/packages/ BEST/vignettes/BEST.pdf). Mean differences and odds ratios were both calculated to include $95 \%$ credible intervals.

\section{Results}

The initial search resulted in 7570 studies, of which 5817 were found to be unique (Fig. 1). After excluding 
TABLE 1. Characteristics of included studies

\begin{tabular}{|c|c|c|c|c|}
\hline Authors \& Year & Design & Surgery Type & Inpatient & Outpatient \\
\hline Silvers et al., 1996 & RCS & ACDF & 53 & 50 \\
\hline Stieber et al., 2005 & RCS & ACDF & Inpatient 1: 30; inpatient 2: 30 & 30 \\
\hline Best \& Sasso, 2007 & RCS & Lumbar decompression in patients over 65 yrs & 30 & 233 \\
\hline Liu et al., 2009 & RCS & ACDF & 64 & 45 \\
\hline Walid et al., 2010 & RCS & ACDF, LMD, \& LDF & 578 & 97 \\
\hline Wohns, 2012 & RCS & Lumbar fusion & 102 & 85 \\
\hline Pugely et al., 2013 & RCS & Lumbar discectomy (single level) & 2658 & 1652 \\
\hline Baird et al., $2014^{4}$ & RCS & Cervical spine & 170,974 & 21,533 \\
\hline Bekelis et al., 2014 & RCS & Lumbar discectomy & 102,592 & 47,125 \\
\hline Martin et al., 2014 & RCS & 1-level ACDF & 2317 & 597 \\
\hline Missios et al., 2014 & RCS & Spinal cord stimulators & 4197 & 4843 \\
\hline McGirt et al., 2015 & RCS & ACDF & 6120 & 1168 \\
\hline Ortega et al., 2015 & RCS & IPD for LSS & 182 & 229 \\
\hline Adamson et al., 2016* & RCS & ACDF & $\begin{array}{l}\text { Total: 484; 1-level ACDF: 274; } \\
\text { 2-level ACDF: } 210\end{array}$ & $\begin{array}{c}\text { Total: 1000; 1-level ACDF: 629; } \\
\text { 2-level ACDF: } 365\end{array}$ \\
\hline Chin et al., 2016 & RCS & LLIF & 40 & 30 \\
\hline Smith et al., 2016 & RCS & MIS lumbar fusion & 160 & 873 \\
\hline
\end{tabular}

IPD for LSS = interspinous process decompression for lumbar spinal stenosis; LDF = lumbar decompression and fusion; LLIF = lateral lumbar interbody fusion; LMD = lumbar microdiscectomy; MIS = minimally invasive surgery; RCS = retrospective cohort study.

* Because this study reported the results of 1-level and 2-level ACDF separately, it was treated as 2 separate studies in the meta-analysis.

those studies that were not looking at human spine surgeries (5754) and those not comparing inpatient and outpatient cohorts for the same surgery (45), we were left with 18 studies that compared inpatient and outpatient spine surgeries. Two of these were further deemed unsuitable for use in the meta-analysis because they did not report data equally for the inpatient and outpatient groups. Thus, the final group of studies for inclusion were the 16 studies described in Table 1.

The number of patients included in the studies ranged from 70 to 192,507. All of the included 16 studies were retrospective cohort studies and are further described in Table 1. For those studies performing further analysis on matched subgroups, only the unmatched groups and corresponding data were used in the meta-analysis. Adamson et al. reported results separately for 1-level and 2-level ACDF surgeries, and therefore their study was treated as if it were 2 separate studies for the purpose of the metaanalysis. ${ }^{1}$ In total, data from 370,195 patients $(290,611$ inpatients and 79,584 outpatients) were used in the analysis. The characteristics of the patients included in the analysis are summarized in the Supplemental Table.

\section{Patient Selection \\ Age}

Thirteen studies (counting the 1-level and 2-level cohorts described by Adamson et al., which we evaluated independently, as separate studies) reported mean ages of both the inpatient and outpatient cohorts. $1,6,9,14,15,17-19,24,25$, ${ }_{27,28}$ Eleven of these studies reported greater mean ages for the patients undergoing procedures on an inpatient basis. A total of 119,554 inpatients and 57,763 outpatients were included in the analysis. Patients undergoing an outpatient procedure were younger than those undergoing an inpatient procedure (mean difference [MD] $-2.34,95 \%$ credible interval $[\mathrm{CrI}]-4.39$ to -0.34 ) (Fig. 2).

\section{Diabetes Mellitus}

Six of the studies reported rates of diabetes mellitus (DM) in their patient cohorts; all of these studies reported higher rates of DM for those undergoing inpatient spine surgeries. ${ }^{1,14,15,17,24,27}$ A total of 9723 inpatient and 3774 outpatient procedures were analyzed. In total, $13.8 \%$ of inpatient cases involved patients with DM, compared to $12.6 \%$ of outpatient cases. Overall, the odds of undergoing an outpatient surgery are about one-third less for those who have DM compared to those who do not (odds ratio [OR] 0.78, 95\% CrI 0.54-0.97) (Fig. 3).

Additional patient selection variables were also reported but did not yield overall significant differences in pooled analysis. Thirteen studies reported sex differences among outpatient and inpatient spine surgeries, and 8 of these studies reported that women were less likely to undergo outpatient spine surgery. Nine studies captured body mass index (BMI), either mean values or as a measure of obesity, and 8 of these studies reported that those with higher BMI values were more likely to be treated in the inpatient setting. Ten of the studies used various measures of comorbidities to include rates of chronic obstructive pulmonary disease (COPD), hypertension, coronary artery disease, and the CCI. All 4 studies reporting a CCI found that undergoing inpatient spine surgery was associated with a higher CCI.

\section{Outcomes}

In addition to looking at patient selection criteria, all 16 
Mundell et al.

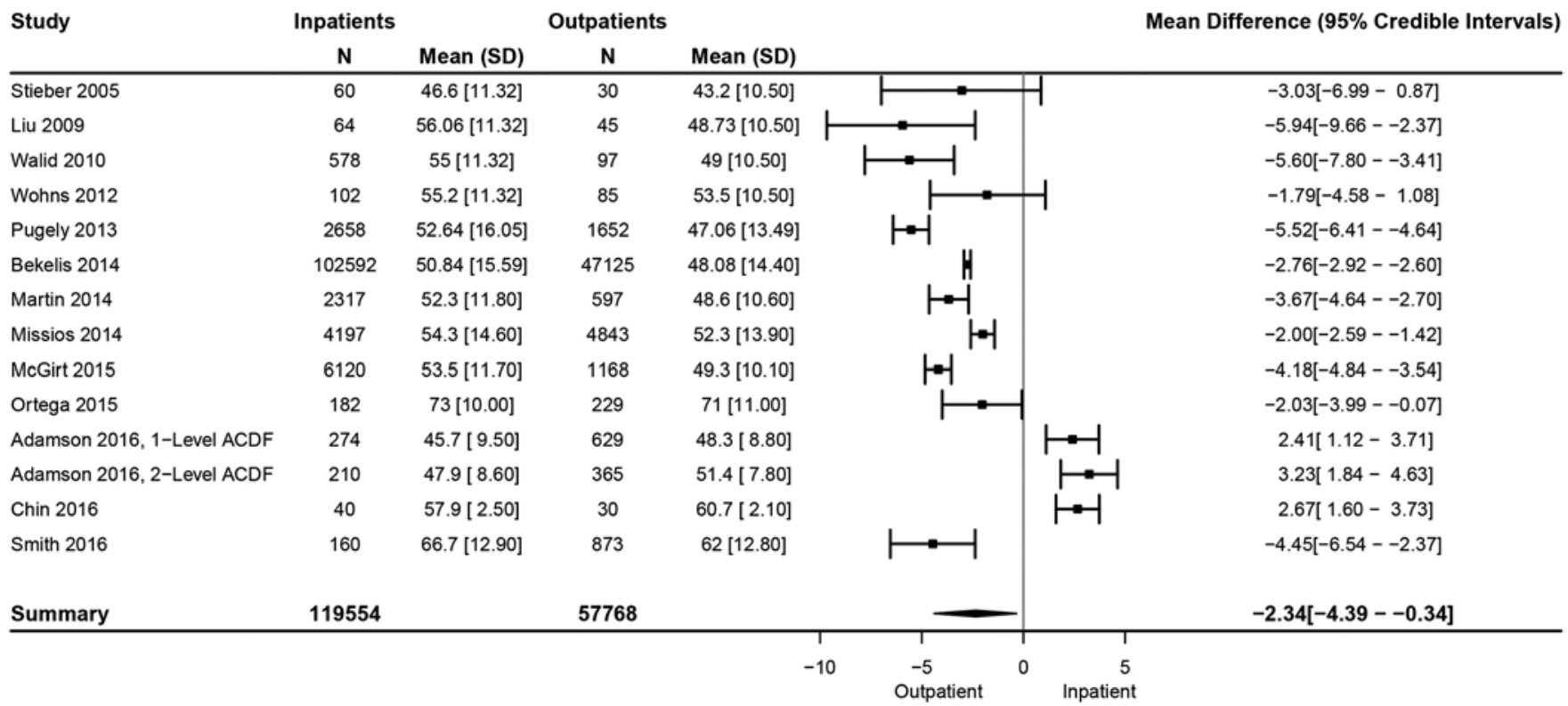

FIG. 2. Forest plot for age as a factor for patient selection between inpatient and outpatient spine surgery.

studies quantified relationships between outcomes, both surgical and financial, and surgical setting. Two studies looked at functional outcomes and found improved outcomes for those undergoing outpatient spine surgeries, ${ }^{9,23}$ Mean length of stay (LOS) was reported by 3 studies, but it was not analyzed due to the fact that outpatient surgeries, based on their definition, should inherently have a shorter LOS. ${ }^{10,16,17}$ All other outcomes measured were found in more than 2 studies and were used in the meta-analysis.

\section{Reoperation Rate}

Six of the studies examined the relationship between surgical setting and 30-day reoperation rates, with all reporting lower reoperation rates for outpatient spine surgeries. ${ }^{1,15,19,21,23}$ In the pooled analysis, undergoing an outpatient spine surgery was associated with a reduction of almost $60 \%$ in the odds of undergoing a reoperation (OR 0.42, 95\% CrI 0.16-0.80) (Fig. 4).

\section{0-Day Readmission Rate}

Data from a total of 30,782 patients in 5 studies were used to look at the relationship between spine surgery setting and 30-day readmission rates. . $^{1,6,18,25,27}$ All 5 studies reported lower readmission rates for patients undergoing outpatient procedures. Similar to the trends found with reoperation rates, undergoing an outpatient procedure was associated with a $60 \%$ reduction in the odds of being readmitted to the hospital following the surgery (OR 0.39, 95\% CrI 0.16-0.74) (Fig. 5).

\section{Complication Rate}

Thirteen of the studies reported the relationship be-

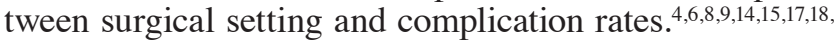
$21,23,25,27,28$ The granularity with which complications were measured varied between studies, but all separated minor complications from major complications (including mortality). All 13 studies found that outpatient surgeries

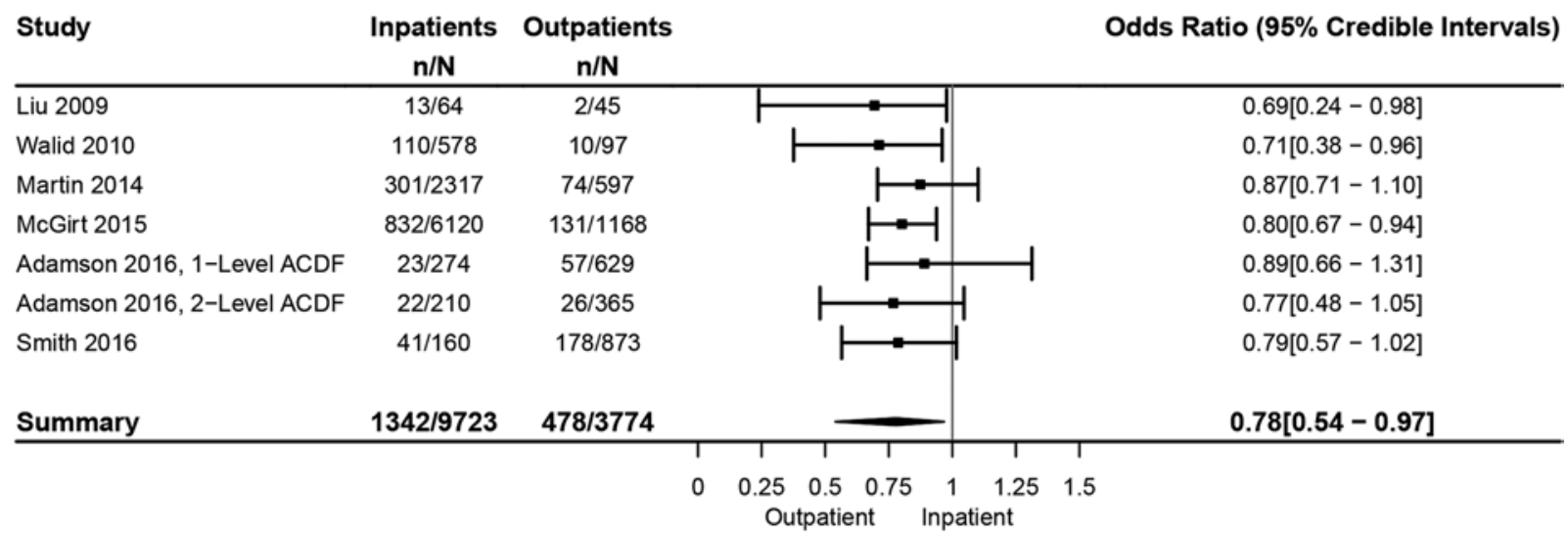

FIG. 3. Forest plot for effect of diabetes on spine surgery setting. 


\begin{tabular}{|c|c|c|c|}
\hline Study & $\begin{array}{c}\text { Inpatients } \\
\mathrm{n} / \mathrm{N}\end{array}$ & $\begin{array}{c}\text { Outpatients } \\
n / N\end{array}$ & Odds Ratio (95\% Credible Intervals) \\
\hline Silvers 1996 & $5 / 53$ & $2 / 50$ & $0.46[0.12-1.16]$ \\
\hline Pugely 2013 & $64 / 2658$ & $29 / 1652$ & $0.66[0.41-1.02]$ \\
\hline Martin 2014 & $32 / 2317$ & $2 / 597$ & $0.32[0.02-0.66]$ \\
\hline McGirt 2015 & $122 / 6120$ & $4 / 1168$ & $0.26[0.09-0.50]$ \\
\hline Ortega 2015 & $42 / 182$ & $33 / 229$ & $0.54[0.33-0.85]$ \\
\hline Adamson 2016, 1-Level ACDF & $1 / 274$ & $1 / 629$ & $0.49[0.10-1.47]$ \\
\hline Adamson 2016, 2-Level ACDF & $1 / 210$ & $1 / 365$ & $0.52[0.10-1.63]$ \\
\hline Summary & $267 / 11814$ & $72 / 4690$ & $0.42[0.16-0.80]$ \\
\hline
\end{tabular}

FIG. 4. Forest plot for comparison of reoperation rates between inpatient and outpatient spine surgery.

were associated with lower complication rates and mortality rates for those reporting them. In total, undergoing an outpatient surgery was associated with a $70 \%$ reduction in the odds of having a complication (OR $0.29,95 \% \mathrm{CrI}$ $0.15-0.50)$. In an attempt to reduce some of the selection differences between the outpatient and inpatient populations, we also performed a meta-regression for complication rates. Unfortunately, only 9 studies had sufficient selection data-i.e., age and results were similar to those found in the aforementioned meta-analysis (OR 0.28, 95\% CrI 0.14-0.50) (Fig. 6).

\section{Cost}

Four of the studies compared costs for outpatient and inpatient spine surgeries, with all reporting significantly lower costs for outpatient procedures. ${ }^{11,15,16,21}$ Overall, outpatient spine surgeries were less costly than inpatient procedures (MD $-\$ 121,392.72,95 \% \mathrm{CrI}-\$ 216,824.81$ to $-\$ 23,632.92$ ) (Fig. 7). This is not surprising, as outpatient surgeries inherently require less time in the hospital and therefore fewer services and less testing during the initial hospitalization. In one study, the authors (Ortega et al.) did look at 18-month costs following inpatient and outpatient interspinous process decompression device placement for lumbar stenosis and found that cost differences were negligible. ${ }^{19}$ Due to this discrepancy in cost reporting, 2 meta- analysis models were run: one with initial costs and one including 18-month costs from Ortega et al. The cost difference remained regardless of the cost data used.

The mean difference and distribution of cost data from the above meta-analysis was then used to assess the difference in direct costs between inpatient and outpatient $\mathrm{ACDF}$ and lumbar laminectomy surgeries in the CDB/RM dataset. It is important to note that $\mathrm{CDB} / \mathrm{RM}$ uses the wellaccepted cost-to-charge ratio to standardize costs across patients, payers, and hospitals. Because selection on age appeared to be consistent across all studies, cost differences were analyzed for a younger age group (30-35 years) and an older age group (65-70 years). While direct costs were lower for outpatient procedures in both subgroups, they were only lower by $\$ 555$ for those age 30-35 years (95\% CrI $-\$ 733$ to $-\$ 374)$, while those age $65-70$ years had a larger cost difference of $\$ 7290$ (95\% CrI $-\$ 7380$ to -\$7190).

\section{Discussion}

To the best of our knowledge, this meta-analysis is the first to perform a comprehensive analysis of the differences in outpatient and inpatient spine surgeries. We also used a novel approach to attempt to validate the meta-analysis results on cost differences using a Bayesian approach to es-

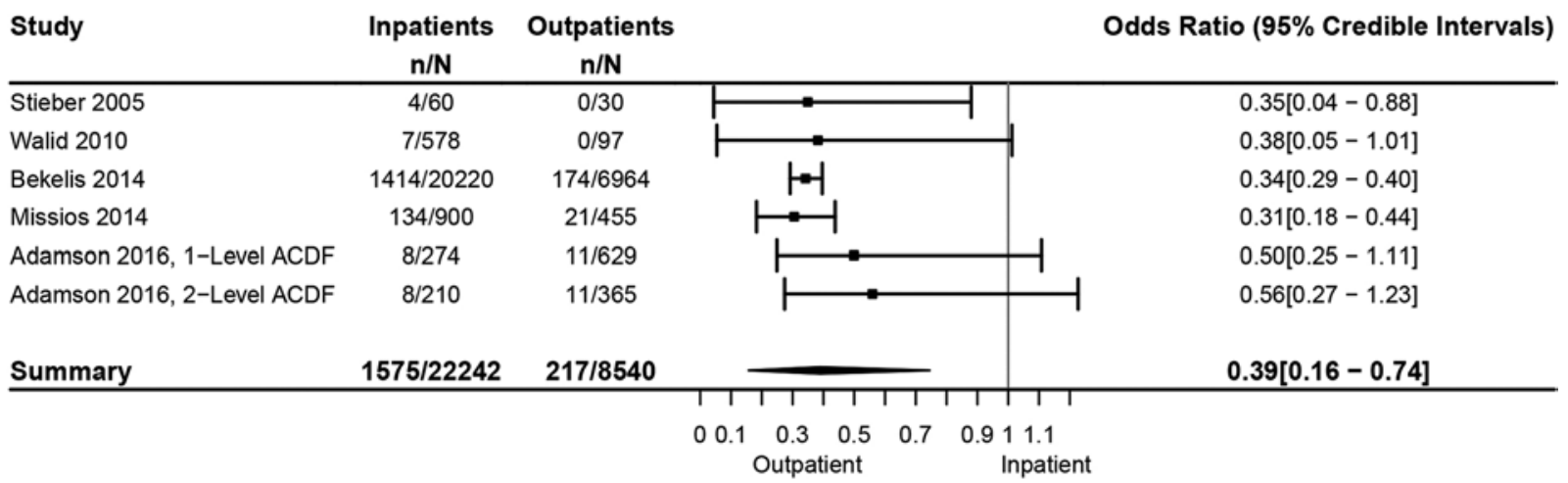

FIG. 5. Forest plot for comparison of 30-day readmission rates between inpatient and outpatient spine surgery. 


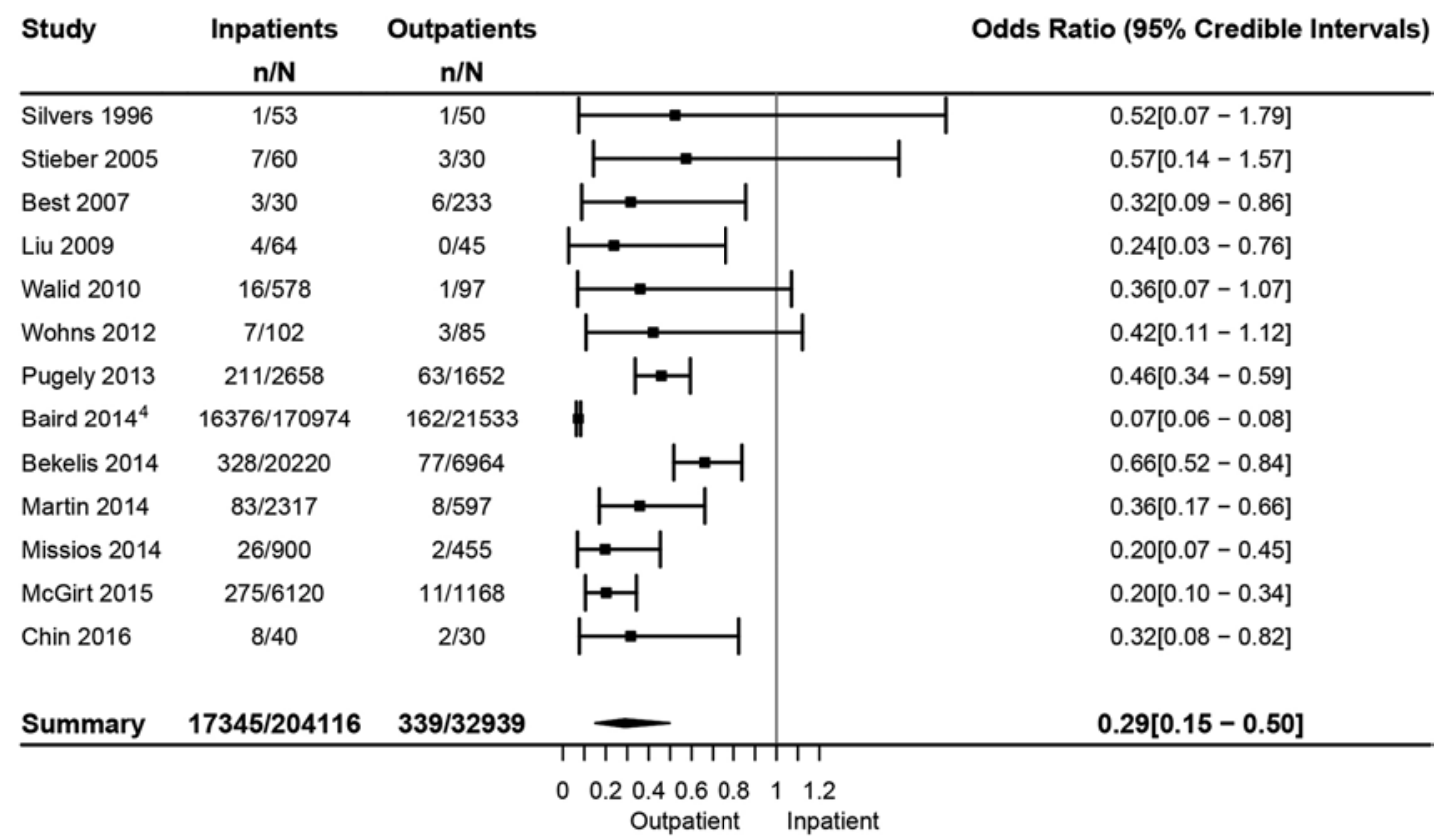

FIG. 6. Forest plot for comparison of complication rates between inpatient and outpatient spine surgery.

timate cost differences in a national dataset. Prior reviews have looked specifically at ACDF surgeries and have found that performing this procedure in the outpatient setting on the correct subset of patients is safe and cost-effective. ${ }^{13,16}$ We found that those patients undergoing outpatient surgeries tended to be younger and were less likely to have DM. Qualitatively, the outpatient surgery patients were also more likely to be male and have a lower number of comorbidities. This is similar to selection criteria reported in reviews of ACDF surgeries and, to a lesser extent, outpatient orthopedic surgical procedures. ${ }^{10,13,16}$ Considering the probable selection bias that exists when looking at outpatient spine surgery outcomes, it is difficult to know how to best interpret the improved outcomes that seem to be associated with the outpatient setting. Two of the studies included in this review attempted to reduce this bias by using propensity score matching between inpatients and outpatients and still found that the perceived benefits of outpatient spine surgery persisted..$^{15,17}$ Unfortunately, propensity score matching is not without flaws and even when well executed cannot fully reduce selection bias (see https://gking. harvard .edu/publications/why-propensity-scores-shouldnot-be-used-formatching), 5,20
We also found that outpatient spine procedures generally appear to result in fewer complications, fewer reoperations, and decreased initial costs. These findings are not unique to the spine surgery arena; others have noted similar findings associated with orthopedic surgery outpatient procedures..$^{10}$ Our additional analysis using the results of the meta-analysis and Bayesian techniques suggests that the cost reductions that come from outpatient procedures may be overstated or at least not uniform across age groups. Younger patients, as a group, tend to have fewer comorbidities than older patients and therefore are less susceptible to patient selection criteria when determining which spine surgical setting is most appropriate for the patient. It appears that patient selection is less of a factor among younger individuals in our additional analysis due to the reduced outpatient cost savings within this group compared to the more heterogeneous older group of patients.

Only one study looked at long-term outcomes and found that the benefits of outpatient surgery in the setting of interspinous process device placement were negligible. ${ }^{19}$ Given the likelihood of selection bias for outpatient spine procedures, there is a high probability that the benefits of outpatient spine surgery in our analysis and past reviews

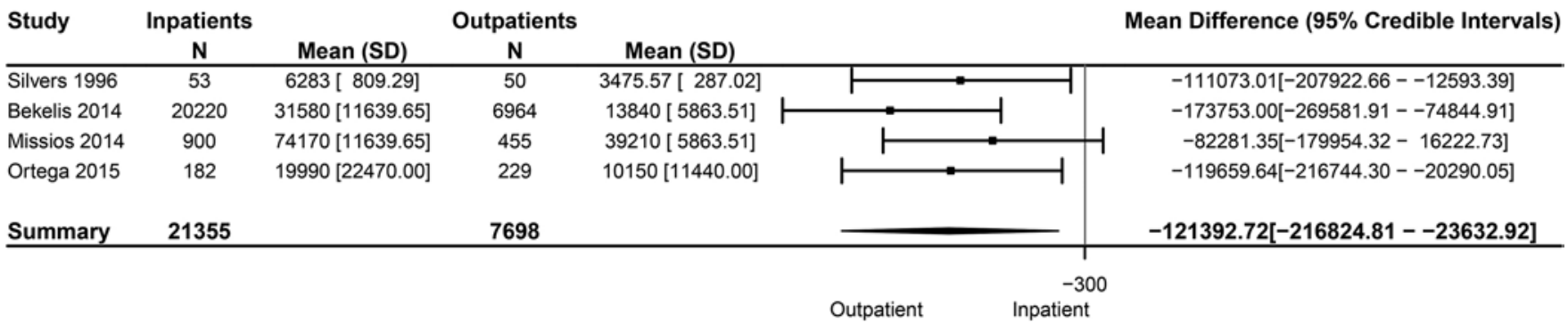

FIG. 7. Forest plot for comparison of costs between inpatient and outpatient spine surgery. 
stem from healthier, younger, and lower-risk patients being offered an outpatient procedure.

\section{Study Limitations}

Our study, while comprehensive in its review, is not without limitations. First, all of the included studies were retrospective studies, which can lead to selection bias in the reported outcomes. This bias is evident in some of the differences seen in patient selection criteria for those chosen to undergo outpatient versus inpatient spine surgeries. Ideally, randomized controlled trials would exist in this arena and would provide an additional perspective on the effects of patient selection on reported outcomes. Second, there was quite a bit of variability in both patient selection variables and outcomes across the studies. This made it difficult to include the maximum number of studies in each analysis. Third, a few of the studies that consistently reported patient selection and outcome data were several orders of magnitude larger than many of the other studies, thereby having a large influence on the pooled effects. Fourth, the reporting of functional outcomes, such as the Oswestry Disability Index, Neck Disability Index, or pain scale scores, varied widely between the included studies; therefore, these measures could not be analyzed in the meta-analysis. Finally, publication bias likely drove many of our results, as those with positive findings-i.e., outpatient is superior to inpatient surgery-are more likely to be published.

\section{Conclusions}

Across the portfolio of spine surgeries, those performed in the outpatient setting, compared to those in the inpatient setting, were associated with more favorable shortterm outcomes and an initial reduction in direct costs. Yet there tends to be a selection bias for outpatient procedures toward younger, healthier patients, which may confound these outcomes. The additional analysis of the national database suggests that cost savings in the outpatient setting may be a result of outpatient procedures being offered more frequently to younger and healthier individuals. To the best of our knowledge, this is a novel approach for validating reported differences in outcomes from a metaanalysis that compares options for surgical management of a disease. Future studies should focus on quantifying outcomes and cost reductions beyond 90 days and reducing or correcting for the selection bias that likely has a significant role in the favorable outcomes associated with outpatient spine surgery in our study.

\section{References}

1. Adamson T, Godil SS, Mehrlich M, Mendenhall S, Asher AL, McGirt MJ: Anterior cervical discectomy and fusion in the outpatient ambulatory surgery setting compared with the inpatient hospital setting: analysis of 1000 consecutive cases. J Neurosurg Spine 24:878-884, 2016

2. Ahn J, Massel DH, Mayo BC, Hijji FY, Narain AS, Aboushaala K, et al: The utility of routinely obtaining postoperative laboratory studies following a minimally invasive transforaminal lumbar interbody fusion. Clin Spine Surg 30:E1405-E1410, 2017

3. Baird EO, Brietzke SC, Weinberg AD, McAnany SJ, Qureshi
SA, Cho SK, et al: Ambulatory spine surgery: a survey study. Global Spine J 4:157-160, 2014

4. Baird EO, Egorova NN, McAnany SJ, Qureshi SA, Hecht AC, Cho SK: National trends in outpatient surgical treatment of degenerative cervical spine disease. Global Spine J 4:143-150, 2014

5. Baser O: Too much ado about propensity score models? Comparing methods of propensity score matching. Value Health 9:377-385, 2006

6. Bekelis K, Missios S, Kakoulides G, Rahmani R, Simmons $\mathrm{N}$ : Selection of patients for ambulatory lumbar discectomy: results from four US states. Spine J 14:1944-1950, 2014

7. Best MJ, Buller LT, Eismont FJ: National trends in ambulatory surgery for intervertebral disc disorders and spinal stenosis: a 12-year analysis of the National Surveys of Ambulatory Surgery. Spine (Phila Pa 1976) 40:1703-1711, 2015

8. Best NM, Sasso RC: Outpatient lumbar spine decompression in 233 patients 65 years of age or older. Spine (Phila Pa 1976) 32:1135-1140, 2007

9. Chin KR, Pencle FJR, Coombs AV, Brown MD, Conklin KJ, O'Neill AM, et al: Lateral lumbar interbody fusion in ambulatory surgery centers: patient selection and outcome measures compared with an inhospital cohort. Spine (Phila Pa 1976) 41:686-692, 2016

10. Crawford DC, Li CS, Sprague S, Bhandari M: Clinical and cost implications of inpatient versus outpatient orthopedic surgeries: a systematic review of the published literature. Orthop Rev (Pavia) 7:6177, 2015

11. Hozo SP, Djulbegovic B, Hozo I: Estimating the mean and variance from the median, range, and the size of a sample. BMC Med Res Methodol 5:13, 2005

12. Kruschke JK: Bayesian estimation supersedes the $t$ test. J Exp Psychol Gen 142:573-603, 2013

13. Lee MJ, Kalfas I, Holmer H, Skelly A: Outpatient surgery in the cervical spine: is it safe? Evid Based Spine Care J 5:101-111, 2014

14. Liu JT, Briner RP, Friedman JA: Comparison of inpatient vs. outpatient anterior cervical discectomy and fusion: a retrospective case series. BMC Surg 9:3, 2009

15. Martin CT, Pugely AJ, Gao Y, Mendoza-Lattes S: Thirtyday morbidity after single-level anterior cervical discectomy and fusion: identification of risk factors and emphasis on the safety of outpatient procedures. J Bone Joint Surg Am 96:1288-1294, 2014

16. McClelland S III, Oren JH, Protopsaltis TS, Passias PG: Outpatient anterior cervical discectomy and fusion: A metaanalysis. J Clin Neurosci 34:166-168, 2016

17. McGirt MJ, Godil SS, Asher AL, Parker SL, Devin CJ: Quality analysis of anterior cervical discectomy and fusion in the outpatient versus inpatient setting: analysis of 7288 patients from the NSQIP database. Neurosurg Focus 39(6):E9, 2015

18. Missios S, Rahmani R, Bekelis K: Spinal cord stimulators: socioeconomic disparities in four US states. Neuromodulation 17:451-456, 2014

19. Ortega A, Sarmiento JM, Patil C, Mukherjee D, Ugiliweneza B, Nuño M, et al: Comparative analysis of inpatient and outpatient interspinous process device placement for lumbar spinal stenosis. J Neurol Surg A Cent Eur Neurosurg 76:443-450, 2015

20. Peikes DN, Moreno L, Orzol SM: Propensity score matching. A note of caution for evaluators of social programs. Am Stat 62:222-231, 2008

21. Pugely AJ, Martin CT, Gao Y, Mendoza-Lattes SA: Outpatient surgery reduces short-term complications in lumbar discectomy: an analysis of 4310 patients from the ACS-NSQIP database. Spine (Phila Pa 1976) 38:264-271, 2013

22. Samuel AM, Grauer JN, Rihn JA, Labrum JT IV: Two-level anterior cervical discectomy and fusion: an outpatient surgery? J Spinal Disord Tech 28:349-351, 2015 
23. Silvers HR, Lewis PJ, Suddaby LS, Asch HL, Clabeaux DE, Blumenson LE: Day surgery for cervical microdiscectomy: is it safe and effective? J Spinal Disord 9:287-293, 1996

24. Smith WD, Wohns RNW, Christian G, Rodgers EJ, Rodgers WB: Outpatient minimally invasive lumbar interbody: fusion predictive factors and clinical results. Spine (Phila Pa 1976) 41 (Suppl 8):S106-S122, 2016

25. Stieber JR, Brown K, Donald GD, Cohen JD: Anterior cervical decompression and fusion with plate fixation as an outpatient procedure. Spine J 5:503-507, 2005

26. Sutton AJ, Abrams KR: Bayesian methods in meta-analysis and evidence synthesis. Stat Methods Med Res 10:277-303, 2001

27. Walid MS, Robinson JS III, Robinson ERM, Brannick BB, Ajjan M, Robinson JS Jr: Comparison of outpatient and inpatient spine surgery patients with regards to obesity, comorbidities and readmission for infection. J Clin Neurosci 17:1497-1498, 2010

28. Wohns RN: A comparison of the safety of lumbar fusions performed as outpatient ( $<25$-hour discharge) or inpatient ( $>24$-hour discharge) procedures. Spine J 12 (9 Suppl):S155-S156, 2012 (Abstract)

\section{Disclosures}

Dr. Nassr reports support for the study described from Pfizer and Premia Spine and fellowship support from AOSpine.

\section{Author Contributions}

Acquisition of data: Mundell, Kerezoudis. Analysis and interpretation of data: Mundell, Kerezoudis. Drafting the article: Bydon, Mundell, Gates, Alvi. Critically revising the article: Bydon, Gates, Kerezoudis, Alvi, Freedman, Nassr, Hohmann. Reviewed submitted version of manuscript: Bydon, Alvi, Freedman, Nassr, Hohmann. Approved the final version of the manuscript on behalf of all authors: Bydon. Statistical analysis: Mundell, Kerezoudis. Study supervision: Bydon.

\section{Supplemental Information}

\section{Online-Only Content}

Supplemental material is available with the online version of the article.

Supplemental Table. https://thejns.org/doi/suppl/10.3171/2018. 4.SPINE1864.

\section{Previous Presentations}

The abstract for this manuscript was presented on March 16, 2018, at the AANS/CNS Joint Section on Disorders of the Spine and Peripheral Nerves Annual Meeting, Orlando, Florida, and also at the Southern Neurosurgical Society Annual Meeting on March 2, 2018, in Marco Island, Florida.

\section{Correspondence}

Mohamad Bydon: Mayo Clinic, Rochester, MN. bydon. mohamad@mayo.edu. 